\title{
An Entropy-Based Class Assignment Detection Approach for RDF Data
}

\author{
Molood Barati ${ }^{1}$, Quan Bai ${ }^{1}$ and Qing Liu $^{2}$ \\ 1 Auckland University of Technology, New Zealand, \\ 2 Data61, CSIRO, Australia \\ mbarati@aut.ac.nz; quan.bai@aut.ac.nz; q.liu@csiro.au
}

\begin{abstract}
The RDF-style Knowledge Bases usually contain a certain level of noises known as Semantic Web data quality issues. This paper has introduced a new Semantic Web data quality issue called Incorrect Class Assignment problem that shows the incorrect assignment between instances in the instance-level and corresponding classes in an ontology. We have proposed an approach called CAD (Class Assignment Detector) to find the correctness and incorrectness of relationships between instances and classes by analyzing features of classes in an ontology. Initial experiments conducted on a dataset demonstrate the effectiveness of CAD.
\end{abstract}

Keywords: Semantic Web data quality issue, Ontology refinement, Incorrect assignment, Knowledge Discovery.

\section{Introduction}

Recently, researchers are tackling with SW data quality issues for refining and reengineering RDF-style Knowledge Bases (KBs). In this paper, we have identified a new SW data quality issue called Incorrect Class Assignment (ICA) problem that shows incorrect assignment between instances in the instance-level and corresponding classes in ontology. The DBpedia ontology defines a Royal class with two subclasses of BritishRoyalty and PolishKing for all royalties. There exist some instances that are incorrectly assigned to unrelated classes in ontology. For example, John I Albert, king of Poland, has been assigned to BritishRoyalty class instead of PolishKing class defined in the DBpedia ontology. This example can be described as an incorrect assignment issue between instance-level data and corresponding classes in ontology. The research problem used in this paper has been modelled in Fig. 1. We name all instances which have been correctly assigned to corresponding classes in ontology as CA. The data quality issue can be defined as the Incorrect Class Assignment problem (ICA) where at least one instance has been incorrectly assigned to class A instead of class B. Under this motivation, we proposed an approach called Class Assignment Detector (CAD) to deal with ICA problem. The main contribution of this paper is threefold including (I) identifying and defining a new SW data quality issue called Incorrect Class Assignment problem (ICA), (II) proposing CAD approach to detect the 
correctness and incorrectness of relationships between instances and classes in ontology by analyzing features of classes, and (III) conducting initial experiments over DBpedia dataset 3.8 to show the effectiveness of CAD.



Fig. 1. Modeling the ICA problem.

This paper is structured as follows. Section 2 reviews related work on SW data quality issues. Sections 3 describes the CAD approach. Section 4 reveals the experimental results. Section 5 explains the conclusion and future work.

\section{Related work}

There exist various forms of SW data quality issues such as missing type prediction, incorrect or incomplete statements, invalid links to external resources, missing link prediction, etc. Studies based on first issue aim to predict missing rdf:type relation in the KBs [1][2]. Second issue refers to the incorrect or incomplete statements of SW data [3][4]. Consider an RDF triple (Rodrigo_Salinas, birthPlace, Puebla_F.C.) shared by DBpedia. The DBpedia provides an invalid object value that is the name of a stadium for Rodrigo Salinas instead of sharing a city or a country name. Third common issue refers to the faulty and invalid links to external RDF-style KBs [5]. Predicting Missing links (i.e. predicates) is another frequent issue in the KBs. Consider Barack Obama as a subject and Honolulu as an object of an RDF triple. Here the question is that how to learn birthPlace relation by mining existing RDF data. A Path ranking Algorithm proposed by [6][7] focused on this issue. To the best of our knowledge, the ICA problem has not been explicitly addressed by the most of existing work.

\section{The architecture of Class Assignment Detector}

The CAD architecture contains two main modules including (1) Class features extraction, and (2) Instance-Class relationship analysis. In Module 1, the CAD extracts features of classes in ontology. The output of Module 1 has been used in Module 2 to assess the correctness and incorrectness of relationships between instances and classes in ontology. 


\subsection{Module 1: Class features extraction}

Generally, a class is a category of things having some common features that make those things distinct from others. To detect the features of classes in our scenario, the initial step is to analyze instance-level data to extract common features among RDF triples. In the following, we first explain the idea behind mining common features from RDF triples. Then, we describe how mining common features of instance-level data leads CAD to extract features of classes.

Identifying common features from RDF triples. The assertion of an RDF triple (i.e., subject, predicate, object) shows a meaningful relationship between a subject and an object provided by a predicate. Considers RDF triples (John I Albert, deathPlace, Poland) and (Casimir III, deathplace, Poland). The subjects of these RDF triples, i.e., John I Albert and Casimir III, have a common feature, i.e., (deathPlace Poland). To extract this behavior from RDF triples, we have defined the concepts of Group Feature and Common Feature as follows.

Definition 1 (Group Feature). Given RDF triples, a Group Feature $g f_{i}$ is a 2-tuple, i.e., $g f_{i}=\left(g_{i}, f_{i}\right) . g_{i}$ is a Group of subjects or objects, i.e., $\left\{s_{1}, s_{2}, \ldots, s_{n}\right\}$ or $\left\{o_{1}, o_{2}, \ldots, o_{n}\right\} . f_{i}$ is a Feature shared by $g_{i}$. Corresponding with the content in $g_{i}, f_{i}$ contains a combination of predicate-object or predicate-subject, i.e., (p, o) or $(\mathrm{p}, \mathrm{s})$.

Definition 2 (Common Feature). Given a Group Feature $g f_{i}=\left(g_{i}, f_{i}\right)$, Feature $f_{i}$ is a Common Feature $c f_{i}$ for Group $g_{i}$, if the number of instances in the $g_{i}$ is greater than or equal to the Minimum Instance Number (MinIN).

Detecting features of classes. RDF-style KBs suffer from ICA problem since publishing SW data is manually maintained by contributors. To this end, CAD takes advantage from information theory [8] to analyze the level of uncertainty from this situation. As explained, a Common Feature shows a common behavior of instances (subjects or objects) in a Group. The information gain allows us to measure which common features are more certain to be used as features of classes in ontology. The following first introduces a measure based on entropy that calculates the uncertainty associated with the whole random space. In the SW, we have defined the entropy of a random space as follows.

Definition 3 (Random Space Entropy). Given an ontology, a Random Space $S$ is a space built up from instances of different classes in ontology. The entropy of $S$ can be calculated by Equation 1:

$$
\operatorname{Entropy}(S)=-\sum_{i=1}^{N} p\left(c_{i}\right) \log _{2} p\left(c_{i}\right)
$$

where $N$ is the total number of classes in the ontology and $p\left(c_{i}\right)=\frac{\left|I n s_{c_{i}}\right|}{|I N S|}$ is the probability of Class $c_{i}$ in the Random Space $S .\left|I n s_{c_{i}}\right|$ is the total number of instances of Class $c_{i} .|I N S|$ is the total number of instances in the Random Space $S$. 
In the information theory, more information can be obtained by a random space with lower entropy, and vice versa. In our scenario, a Common Feature can be shared by instances of different classes in ontology. Therefore, the information gained from a Common Feature depends on the types of instances in its Group. Few types can cause lower entropy and consequently more information gained from the Common Feature. By relying on the fact, we have defined the concepts of Common Feature Information and Common Feature Space as follows.

Definition 4 (Common Feature Information). Given a Random Space $S$ and a Common Feature $c f_{i}$, the information gained from $c f_{i}$ is a normalized value measured by Equation 2:

$$
\operatorname{NormGain}\left(S, c f_{i}\right)=\frac{\operatorname{Entropy}(S)-\frac{\left|\operatorname{Ins}_{c f_{i}}\right|}{|I N S|} \operatorname{Entropy}\left(S_{c f_{i}}\right)}{\operatorname{Entropy}(S)}
$$

where $\operatorname{Entropy}(S) \neq 0$ and $0 \leq N \operatorname{NormGain}\left(S, c f_{i}\right) \leq 1$.

Definition 5 (Common Feature Space). Given a Common Feature $c f_{i}$, RDF triples and its corresponding ontology, a Common Feature Space $S_{c f_{i}}$ is a space built up from instances that share the Common Feature $c f_{i}$. The Entropy of Common Feature Space $S_{c f_{i}}$ can be computed by Equation 3:

$$
\operatorname{Entropy}\left(S_{c f_{i}}\right)=-\sum_{i=1}^{n} p\left(c_{i . c f_{i}}\right) \log _{2} p\left(c_{i . c f_{i}}\right)
$$

where $n$ is the total number of classes in the Common Feature Space $S_{c f_{i}}$ and $p\left(c_{i . c f_{i}}\right)=\frac{\left|I n s_{c_{i} . c f_{i}}\right|}{\left|I n s_{c f_{i}}\right|}$ is the probability of class $c_{i}$ in the Common Feature Space $S_{c f_{i} \cdot} \cdot\left|I n s_{c_{i} . c f_{i}}\right|$ is the total number of instances of class $c_{i}$ that share $c f_{i} \cdot\left|I n s_{c f_{i}}\right|$ is the total number of instances that share $c f_{i}$.

According to Equation 2, the more information gained by a Common Feature indicates fewer types in the random space generated by the Common Feature.

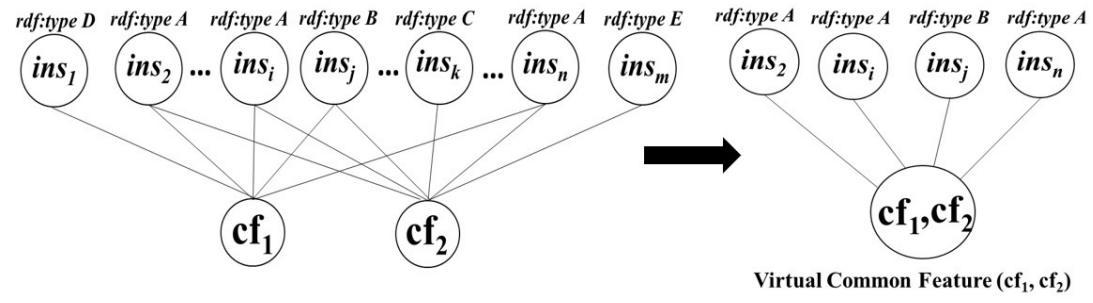

Fig. 2. An example of a Virtual Common Feature.

On the one side, a Common Feature can be shared by instances of different classes. On the other side, instances of a class might share more than one Common Feature. In this paper, we have evaluated the information gained from combinations of common features. Consider Fig. 2 to explain the idea. Common 
Feature $c f_{1}$ is shared by instance $i n s_{1}$ with $r d f$ :type $D$, and $i n s_{2}, i n s_{i}, i n s_{n}$ with rdf:type $A$, and $i n s_{j}$ with $r d f$ :type $B$. Common Feature $c f_{2}$ is also shared by $i n s_{2}$, $i n s_{i}$, ins $s_{n}$ with $r d f:$ type $A$, and $i n s_{k}$ with $r d f: t y p e ~ C$, and $i n s_{m}$ with $r d f: t y p e ~ E$. The combination of $\left(c f_{1}, c f_{2}\right)$ gains more information to compare with each $c f_{1}$ and $c f_{2}$. Because the Random Space of $\left(c f_{1}, c f_{2}\right)$ contains lower entropy and fewer types (i.e., types $A$ and $B$ ) to compare with $c f_{1}$ and $c f_{2}$ that contain instances with $\{$ rdf:type A, rdf:type B, rdf:type D $\}$ and $\{$ rdf:type A, rdf:type $C$, rdf:type E\}, respectively. Based on this motivation, the concept of Virtual Common Feature is defined as follows.

Definition 6 (Virtual Common Feature). A Virtual Common Feature vcf is a combination of $n(n \geq 2)$ common features where the number of instances that share $v c f$ is greater than or equal to MinIN.

A Virtual Common Feature $v c f$ shows there is a number of instances that share more than one Common Feature. In this regard, the information gained from a Virtual Common Feature can be computed by Equations 2 and 3. We just need to replace $\left|I n s_{c f_{i}}\right|$ and Entropy $\left(S_{c f_{i}}\right)$ with $\left|I n s_{v c f_{i}}\right|$ and Entropy $\left(V S_{v c f_{i}}\right)$. The $\left|I n s_{v c f_{i}}\right|$ is the total number of instances that share $v c f_{i}$ and Entropy $\left(V S_{v c f_{i}}\right)$ is the Entropy of Virtual Common Feature Space.

Given a Common Feature $c f_{i}$ and a Virtual Common Feature $v c f_{i}$, the more information indicates lower entropy (i.e., lower uncertainty) in the random spaces generated by $c f_{i}$ and $v c f_{i}$. This fact reveals that most of instances that have shared $c f_{i}$ and $v c f_{i}$ have the same type. Based on this motivation, the concept of Class Feature is defined as follows.

Definition 7 (Class Feature). A Common Feature $c f_{i}$ or a Virtual Common Feature $v c f_{i}$ is a Class Feature for Class $c_{i}$, if the information gained from $c f_{i}$ or $v c f_{i}$ is greater than or equal to NormGain Thresholds $(N G T h)$.

Note that $c f_{i}\left(\right.$ or $\left.v c f_{i}\right)$ is a Class Feature for Class $c_{i}$ where most instances that share $c f_{i}$ have been assigned to Class $c_{i}$. It is important to mention that a class can have multiple class features including common features and virtual common features with information gained greater than or equal to NGTh.

\subsection{Module 2: Instance-Class relationship analysis}

If we take an instance with type and features, the goal of Module 2 is to analyse the correctness (i.e., CA) and incorrectness (i.e., ICA) of relationships between the instance and classes. To this end, Algorithm 1 has been implemented to assess the above targets for a given instance in four different statuses including (I) the CA status of an instance with one Feature, (II) the ICA status of an instance with one Feature, (III) the CA status of an instance with multiple features, and (IV) the ICA status of an instance with multiple features. Algorithm 1 receives Minimum Instance Number (MinIN), NormGain Thresholds (NGTh), classes $(C)$, features of classes (C.features), instances (Iset), and features of instances (I.features) as inputs. Algorithm 1 returns $C A$ and ICA statutes for given instances as an output. Note that the status of ins $_{i}$ is Undecidable if ins $_{i}$ is neither CA nor ICA. 




\section{Experiments and analysis}

The following experiments have been conducted over DBpedia dataset 3.8 that is one of the most common errors encountered RDF-style KBs. By using DBpedia dataset, we considered two classes called Food and Hotel. Each class contains about 750 instances. The goal of following experiments is to check the accuracy of CAD in analyzing the correctness and incorrectness of relationships between instances and classes. Generally, the accuracy of a system is the degree of closeness between a measured value and the true value. In our scenario, a measured value refers to the number of correctly (i.e., CA) and incorrectly (i.e, ICA) assigned instances detected by CAD approach. While a true value indicates the predefined number of $\mathrm{CA}$ and ICA instances in a class. Thus, we correctly assigned 700 instances to the Hotel class that indicates a true value for CA instances. We also incorrectly assigned 50 instances of Hotel class to the Food class that shows a true value for ICA instances. To measure the accuracy of CAD approach, two measurements called Accuracy ${ }_{C A}$ and Accuracy $_{I C A}$ are defined as follows. 
Given a class, the accuracy of CAD in detecting correctly assigned instances can be computed by Equation 4:

$$
\text { Accuracy }_{C A}=\frac{\left|i n s_{C A} \cap I N S_{C A}\right|}{\left|I N S_{C A}\right|}
$$

where $i n s_{C A}$ is the number of correctly assigned instances detected by CAD and $I N S_{C A}$ is a true value for the predefined number of CA instances in the class.

Given a class, the accuracy of CAD in detecting incorrectly assigned instances can be measured by Equation 5:

$$
\text { Accuracy }_{I C A}=\frac{\mid \text { ins }_{I C A} \cap I N S_{I C A} \mid}{\left|I N S_{I C A}\right|}
$$

where ins $_{I C A}$ is the number of incorrectly assigned instances detected by CAD and $I N S_{I C A}$ is a true value for the predefined number of ICA instances in the class.



(a)

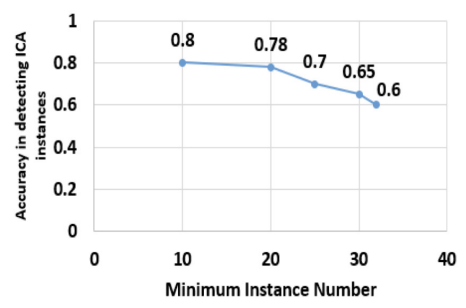

(c)

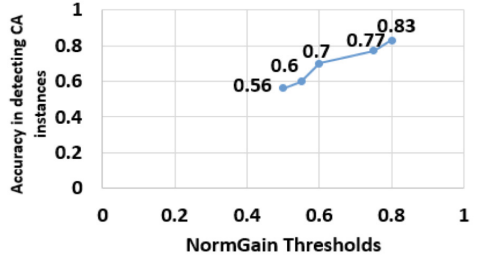

(b)

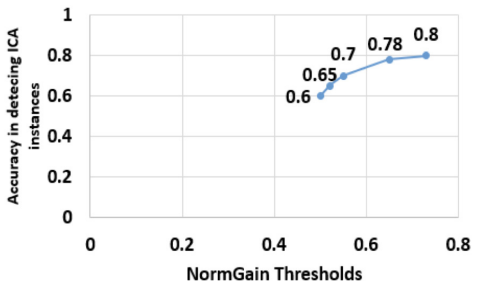

(d)

Fig. 3. (a) Accuracy in detecting CA instances in different MinIN, (b) Accuracy in detecting CA instances in different $N G T h$, (c) Accuracy in detecting ICA instances in different MinIN, (d) Accuracy in detecting ICA instances in different NGTh.

Fig. 3(a) shows that the accuracy of CAD approach in detecting CA instances has been gradually decreased by increasing MinIN. One reason behind such reduction is related to the strategy of selecting common features by using MinIN. Consider the process of analyzing common features in Algorithm 1. Given an instance, if the feature shared by ins $_{i}$ is not a Common Feature, then the status of $i n s_{i}$ is undecidable. For example, an RDF triple (White House, location, 
Herm) detected indicates that White House is an instance of Hotel class with a particular feature i.e., (location, Herm). In the Hotel class, Herm is the only instance that has shared (location, Herm) as a particular feature. Algorithm 1 ignores some instances in case the features shared by them have not identified as common features. Fig. 3(b) shows that the accuracy of CAD approach in detecting CA instances has been grown by increasing NGTh. Fig. 3(c) represents that the accuracy of CAD in detecting ICA instances is reduced by increasing MinI. Consider again the RDF triple (White House, location, Herm). If White House has been incorrectly assigned to the Food class, Algorithm 1 ignores White House since (location, Herm) has not identified as a Common Feature.

\section{Conclusions and future work}

This paper has introduced a new SW data quality issue called Incorrect Class Assignment (ICA) problem that indicates incorrect assignment between instancelevel data and corresponding classes in an ontology. So, we proposed an entropybased approach called Correct Assignment Detector (CAD) to deal with ICA problem. A direction for future work is to apply Natural Language Processing (NLP) techniques on predicates of common features to find out more similar behaviors taken by instances in the groups.

\section{References}

1. A. Melo, J. Völker, and H. Paulheim, "Type prediction in noisy rdf knowledge bases using hierarchical multilabel classification with graph and latent features," International Journal on Artificial Intelligence Tools, vol. 26, no. 02, p. 1760011, 2017.

2. K. Gunaratna, K. Thirunarayan, A. Sheth, and G. Cheng, "Gleaning types for literals in rdf triples with application to entity summarization," in International Semantic Web Conference. Springer, 2016, pp. 85-100.

3. J. Lehmann, D. Gerber, M. Morsey, and A.-C. N. Ngomo, "Defacto-deep fact validation," in International Semantic Web Conference. Springer, 2012, pp. 312-327.

4. G. Töpper, M. Knuth, and H. Sack, "Dbpedia ontology enrichment for inconsistency detection," in Proceedings of the 8th International Conference on Semantic Systems. ACM, 2012, pp. 33-40.

5. J. Volz, C. Bizer, M. Gaedke, and G. Kobilarov, "Discovering and maintaining links on the web of data," in International Semantic Web Conference. Springer, 2009, pp. 650-665.

6. N. Lao and W. W. Cohen, "Relational retrieval using a combination of pathconstrained random walks," Machine learning, vol. 81, no. 1, pp. 53-67, 2010.

7. N. Lao, T. Mitchell, and W. W. Cohen, "Random walk inference and learning in a large scale knowledge base," in Proceedings of the Conference on Empirical Methods in Natural Language Processing. Association for Computational Linguistics, 2011, pp. $529-539$.

8. E. T. Jaynes, "Information theory and statistical mechanics," Physical review, vol. 106, no. 4, p. 620, 1957. 